\title{
Is there any role for positron emission tomography-computed tomography after induction therapy for locally advanced non-small cell lung cancer?
}

\author{
Traves D. Crabtree, MD, ${ }^{\mathrm{a}}$ and Akash Sharma, $\mathrm{MD}^{\mathrm{b}}$
}

\footnotetext{
From the a Division of Cardiothoracic Surgery, Department of Surgery, Washington University School of Medicine, St Louis, Mo; and the ${ }^{\mathrm{b}}$ Division of Nuclear Medicine, Department of Radiology, Washington University School of Medicine, St Louis, Mo.

Disclosures: Authors have nothing to disclose with regard to commercial support.

Received for publication Sept 3, 2015; accepted for publication Sept 3, 2015; available ahead of print Oct 15 , 2015.

Address for reprints: Traves D. Crabtree, MD, Division of Cardiothoracic Surgery, Washington University School of Medicine, Campus Box 8234; 660 S Euclid Ave, St Louis, MO 63110 (E-mail: crabtreet@wudosis.wustl. edu).

J Thorac Cardiovasc Surg 2016;151:911-2

$0022-5223 / \$ 36.00$

Copyright $(2016$ by The American Association for Thoracic Surgery

http://dx.doi.org/10.1016/j.jtcvs.2015.09.025
}

The ultimate goal for utilizing diagnostic imaging modalities is to assist in the planning of treatment, and in counseling the patient regarding their subsequent management and prognosis. One would generally anticipate that an imaging modality such as positron emission tomography (PET) should provide consistent and reliable data that might impact the decision algorithm with respect to the preparation for biopsy, selection of therapy (ie, operative vs nonoperative), planning of surgical strategy, and assessment of treatment response. PET imaging has proved to be a valuable adjunct in the clinical staging of patients with lung cancer. Despite the limitations of PET, it has helped in the identification of occult distant metastases not clearly delineated with other imaging modalities, and it has helped to guide surgical staging techniques such as endobronchial ultrasonography and mediastinoscopy. These benefits have ultimately assisted treating physicians to avoid unnecessary and futile operations in patients with metastatic disease and have facilitated the identification of nodal disease that may benefit from multimodality therapy.

Although our understanding of the relationship between the metabolic activity of lung cancers and the biologic behavior of these cancers is rudimentary, PET has introduced the concept of correlating this activity with prognosis. Still, objective quantification of the degree of tumor metabolic activity has been historically inconsistent because of the multiple factors contributing to wide variation of reported standard uptake value (SUV). Contemporary efforts to standardize fludeoxyglucose F 18 (INN fludeoxyglucose [18F]; FDG) dose, FDG administration, and quantitative assessment of FDG uptake have attempted to address these shortcomings to create reproducible measures of tumor metabolism; however, this variability remains a limitation of PET.

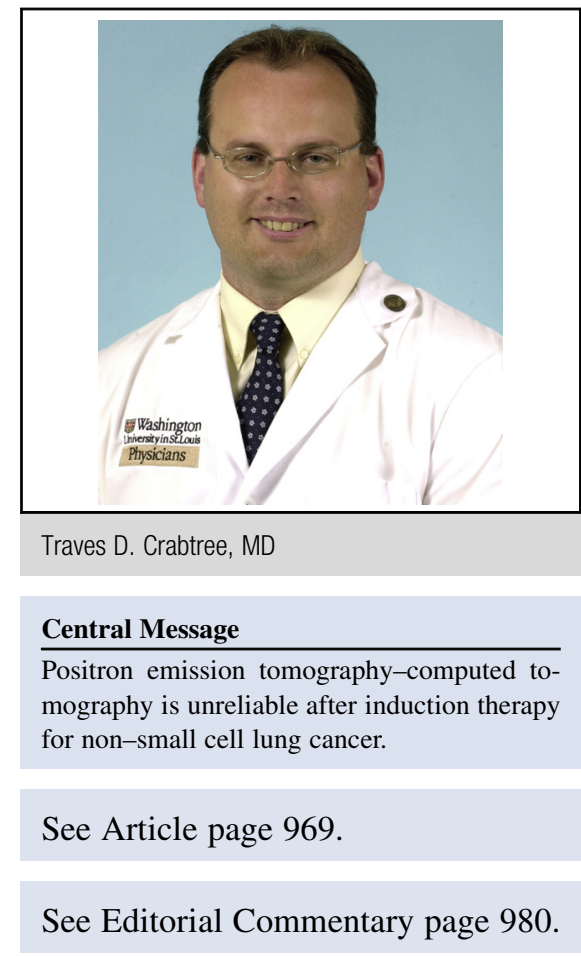

Several publications have attempted to address the prognostic role of PET in NSCLC. A potential correlation between PET SUV of the primary tumor and an increase in the likelihood of occult nodal disease in patients with clinical stage I non-small cell lung cancer (NSCLC) has led to the utilization of primary tumor SUV as a surrogate, independent of tumor size, to guide selection of patients for surgical mediastinal staging in a clinical trial. ${ }^{1}$ Some previous reports have demonstrated a linear relationship with changes in the primary tumor PET SUV before and after induction therapy with pathologic response and overall survival. ${ }^{2,3}$ These results, unfortunately, have always been limited in their clinical application because of a significant number of false-positives and false-negative results.

One appealing utility of PET would be to correlate PET SUV quantitatively with treatment response to chemotherapy or radiotherapy. This conceivably would apply to patients being treated with definitive nonsurgical therapy or those undergoing neoadjuvant therapy before curative resection. The recent investigation by Ripley and colleagues 
from Memorial Sloan Kettering Cancer Center, presented at the 2015 annual meeting of the American Association for Thoracic Surgery and appearing in this issue of the Journal, retrospectively studied 101 patient undergoing induction chemotherapy for clinical stage IIIA NSCLC with mediastinal $\mathrm{N} 2$ disease. ${ }^{4}$ The study design was notable for the fact that $81 \%$ of the patients underwent PET after induction therapy but, regardless of the results, none of the patients underwent mediastinal restaging before resection. Several nodal stations without significant SUV uptake on initial PET became FDG avid after induction therapy. More than half the nodal stations with nonavid mediastinal disease on postinduction PET were seen to have persistent pathologic N2 disease at resection. Among the patients with clinical IIIA disease without pathologic N2 disease after induction chemotherapy and resection, $50 \%$ had residual PET N2 nodal avidity and 50\% did not. As a prognostic tool for survival, relative decrease in the SUV of the primary tumor or lymph nodes, resolution of mediastinal SUV, and sterilization of $\mathrm{N} 2$ disease were not predictive of overall survival. These results affirm the work of others with prohibitive rates of false-positive and false-negative PET findings after induction chemotherapy and chemoradiotherapy.

With regard to the broader use of PET for NSCLC, a recent Cochrane analysis of PET for assessment of N2 and $\mathrm{N} 3$ disease demonstrated an overall sensitivity of $77.4 \%$ and a specificity estimate of $90 \%$, concluding that PET-computed tomography (PET-CT) alone is insufficient to guide treatment decisions. The most concerning aspect of the inconsistency seen in the sensitivity and specificity of PET-CT in this setting was the impact of different scanner types, histologic subtypes, FDG dose, country of origin, and general variability of results. ${ }^{5}$ In addition, others have demonstrated significantly decreased specificity of PET in areas with endemic infectious lung disease such as histoplasmosis. ${ }^{6}$ All this variability impacts the physician's ability to make decisions on the basis of PET results for primary assessment of lung cancer and after induction therapy.

The goal of using PET or other functional modalities in guiding diagnosis and treatment remains as clinically relevant as ever. Although the described studies demonstrated that certain targeted questions such as PET after induction therapy have not been answered adequately by PET, it is also very important to note that performance and reporting of PET studies form a complex process that continues to evolve and that standardization, when possible, is highly desirable. Most researchers interested in the development of tumor-specific markers contend that more specific assessment of metabolism thus achieved would likely improve the specificity of PET and provide more reliable data to guide treatment. Such improvements might help us adjust therapies on the basis of the PET response.
Chemotherapy regimens could be adjusted or the timing of surgical intervention could be altered according to the radiographic assessment of the functional activity of the tumor. Just as interim response to treatment is currently being vigorously investigated in the treatment of lymphoma, a more standardized approach to PET, closely coupled with contrast-enhanced anatomic imaging, with further addition of tracers targeting specific tumors is needed to fulfill the promise of personalized medicine. Only when the imaging modality is standardized in use for a given set of patients can it positively impact patients with lung cancer. This may also apply to multiple other malignancies such as esophageal cancer, pancreatic cancer, and rectal cancer.

PET-CT remains a valuable adjunctive modality for initial staging of NSCLC along with anatomic assessment of disease. Because of the nonspecificity of FDG metabolism, most PET findings still need to be confirmed with pathologic evaluation. In the context of evaluation of disease response after induction therapy, however, there seems to be very little clinical benefit to PET unless the previously mentioned variabilities are addressed. Identification of otherwise unrecognized or new metastatic disease after induction therapy is very uncommon relative to the initial staging PET-CT. With this in mind, and with PET-CT's inability to delineate the treatment response to therapy accurately from posttreatment changes, it is difficult to justify the use of PET in this setting. Furthermore, in an era of increasing demand for cost efficiency and value-based assessment of diagnostic testing, it would seem that this relative cost-benefit ratio of PET-CT precludes routine use of this modality after induction therapy for NSCLC. This study by Ripley and colleagues ${ }^{4}$ brings to light important issues that need to be addressed as we continue to assess the role of PET imaging in the specific context of postinduction therapy.

\section{References}

1. Fernandez FG, Kozower BD, Crabtree TD, Force SD, Lau C, Pickens A, et al. Utility of mediastinoscopy in clinical stage I lung cancers at risk for occult mediastinal nodal metastases. J Thorac Cardiovasc Surg. 2015;149:35-41. 42.e1.

2. Cerfolio RJ, Bryant AS, Winokur TS, Ohja B, Bartolucci AA. Repeat FDGPET after neoadjuvant therapy is a predictor of pathologic response in patients with non-small cell lung cancer. Ann Thorac Surg. 2004;78:1903-9; discussion 1909.

3. Hellwig D, Graeter TP, Ukena D, Georg T, Kirsch C, Schäfers HJ. Value of F-18-fluorodeoxyglucose positron emission tomography after induction therapy of locally advanced bronchogenic carcinoma. J Thorac Cardiovasc Surg. 2004;128:892-9.

4. Ripley RT, Suzuki K, Tan KS, Adusumilli PS, Huang J, Park BJ, et al, Postinduction positron emission tomography assessment of N2 nodes is not associated with ypN2 disease or overall survival in stage IIIA non-small cell lung cancer. J Thorac Cardiovasc Surg. 2016;151:969-79.

5. Schmidt-Hansen M, Baldwin DR, Hasler E, Zamora J, Abraira V, Roqué I, et al. PET-CT for assessing mediastinal lymph node involvement in patients with suspected resectable non-small cell lung cancer. Cochrane Database Syst Rev. 2014;11:CD009519.

6. Deppen SA, Blume JD, Kensinger CD, Morgan AM, Aldrich MC, Massion PP, et al. Accuracy of FDG-PET to diagnose lung cancer in areas with infectious lung disease: a meta-analysis. JAMA. 2014;312:1227-36. 